\title{
Incidence of Immune-Related Adverse Events with Program Death Receptor-1- and Program Death Receptor-1 Ligand-Directed Therapies in Genitourinary Cancers
}

\author{
Benjamin L. Maughan ${ }^{1,2 t}$, Erin Bailey ${ }^{1 t}$, David M. Gill ${ }^{2}$ and Neeraj Agarwal ${ }^{1,2 *}$ \\ ${ }^{1}$ Huntsman Cancer Institute, University of Utah, Salt Lake City, UT, USA, ${ }^{2}$ Department of Internal Medicine, University of \\ Utah, Salt Lake City, UT, USA
}

OPEN ACCESS

Edited by:

Viktor Grünwald,

Hannover Medical School, Germany

Reviewed by:

Christoph Seidel,

University Medical Center Hamburg-

Eppendorf, Germany

Giuseppe Di Lorenzo,

University Federico II of Napoli, Italy

Takeshi Yuasa,

Japanese Foundation for Cancer

Research, Japan

*Correspondence:

Neeraj Agarwal

neeraj.agarwal@hci.utah.edu

tThese authors have contributed equally to this work.

Specialty section:

This article was submitted to

Genitourinary Oncology,

a section of the journal

Frontiers in Oncology

Received: 30 January 2017

Accepted: 14 March 2017

Published: 03 April 2017

Citation:

Maughan BL, Bailey E, Gill DM and

Agarwal N (2017) Incidence of

Immune-Related Adverse Events with

Program Death Receptor-1- and

Program Death Receptor-1

Ligand-Directed Therapies in

Genitourinary Cancers.

Front. Oncol. 7:56.

doi: 10.3389/fonc.2017.00056
Program death receptor-1 (PD-1) and program death receptor-1 ligand (PD-L1) inhibitors are increasingly being used in the clinic to treat a growing number of malignancies, including many genitourinary (GU) malignancies. These immune-based therapies have demonstrated a distinct toxicity profile compared to traditional chemotherapy and the targeted therapies directed at the vascular endothelial growth factor pathway or the mammalian target of rapamycin pathway. Autoimmune toxicity targeting the skin, gastrointestinal tract, or the endocrine organs are some of the more common adverse events (AEs) noted with these therapies. Here in, we report the results of a systematic review of the incidence of toxicities in GU cancers reported in the phase II or phase III clinical trials using single-agent PD-1 or PD-L1 inhibitors. Overall, the rate of serious (grades 3-4) AEs was noted in approximately $15 \%$ of patients. The AEs noted were similar between all the agents tested, highlighting the overall class effect of these therapies. The incidence in GU cancers is similar to those seen in other malignancies. Given the widespread and high volume real-world use of these agents, it is important for oncologists to be familiar with these side effects to minimize the risks for patients while undergoing therapy.

Keywords: immunotherapy, checkpoint blockade, toxicity, treatment, autoimmune, immune-related adverse events

\section{INTRODUCTION}

The approval of ipilimumab for metastatic melanoma in 2011 spearheaded the development of numerous anticancer therapies targeting immune checkpoint pathways. To date, these novel therapies have been approved in quick succession by the US Food and Drug Administration (FDA) for the treatment of a variety of solid tumors and hematologic malignancies. These therapies have been shown to produce prolonged, durable complete remissions in a subset of patients even with high volume metastatic disease, which was not previously seen with traditional chemotherapy or the targeted therapies. The success of ipilimumab, a monoclonal antibody targeting cytotoxic T-lymphocyte-associated protein 4, led to an explosion of research resulting in the development of new immunotherapy agents, focusing on a variety of new immune targets. The most prominent of 
these new discoveries are monoclonal antibodies targeting programmed cell death protein 1 program death receptor-1 (PD-1), for example, nivolumab and pembrolizumab, or its ligand program death receptor-1 ligand (PD-L1), for example, durvalumab and avelumab. Many of these PD checkpoint inhibitors are now FDA approved, leading to a significant increase in their clinical use over the last few years. Through the clinical trials already conducted, it is evident that the side effects are quite distinct from traditional chemotherapy and the targeted therapies previously approved. Here, we report the results of a systematic review on the incidence of autoimmune adverse events (AEs) reported in the phase II or phase III clinical trials in genitourinary (GU) cancers using single-agent PD-1 or PD-L1 inhibitors.

\section{MECHANISM OF ACTION OF PD-1 AND PD-L1 INHIBITORS}

Program death receptor- 1 and PD-L1 are cell surface proteins that are part of the large immunoglobulin superfamily. Antagonists of these receptors are included in the diverse drug class referred to as immune checkpoint inhibitors. The PD-1 and PD-L1 antagonistic antibodies are designed ultimately to augment the immune system as a mechanism of treating cancer. Treatment directed against these targets directly leads to activation of cytotoxic T-lymphocytes (Figure 1). The mechanism of action for checkpoint inhibitors and the clinical efficacy of these therapies in GU and other malignancies have been reviewed previously in detail (1-5). Briefly, activation or suppression of $\mathrm{T}$ cells requires a two-step process. First, the antigen presenting cell (APC), such as dendritic cells, processes and presents the antigen to T-cells via the major histocompatibility complex (MHC) via direct binding to the T-cell receptor (TCR). In addition to the interaction of MHC and TCR, a second co-stimulatory or co-suppressor signal is required for an appropriate immune response. PD-1 is a receptor found on many immune mediator cells such as T-cells, natural killer cells, dendritic cells, and B-cells (6). PD-L1 is one of two known ligands (PD-L1 and PD-L2) for PD-1, and both are co-inhibitory to T-cell activation. PD-L1 is found on APCs as well as tumor cells (7). The co-inhibitory signal from PD-1 and PD-L1 signaling provides the necessary inhibitory signal, driving the T-cell into a state of inactivity.

\section{CLINICAL ACTIVITY OF PD-1 OR PD-L1 CHECKPOINT INHIBITORS IN GU CANCERS}

These agents have demonstrated significant activity in GU malignancies including renal cell carcinoma $(8,9)$ and urothelial carcinoma, leading to approval of some of these agents (10). Additionally, trials investigating many of these agents in the advanced prostate cancer are ongoing.

In the seminal report on the efficacy of nivolumab (8), patients with metastatic renal cell carcinoma were randomly assigned in 1:1 fashion to treatment with nivolumab or everolimus after

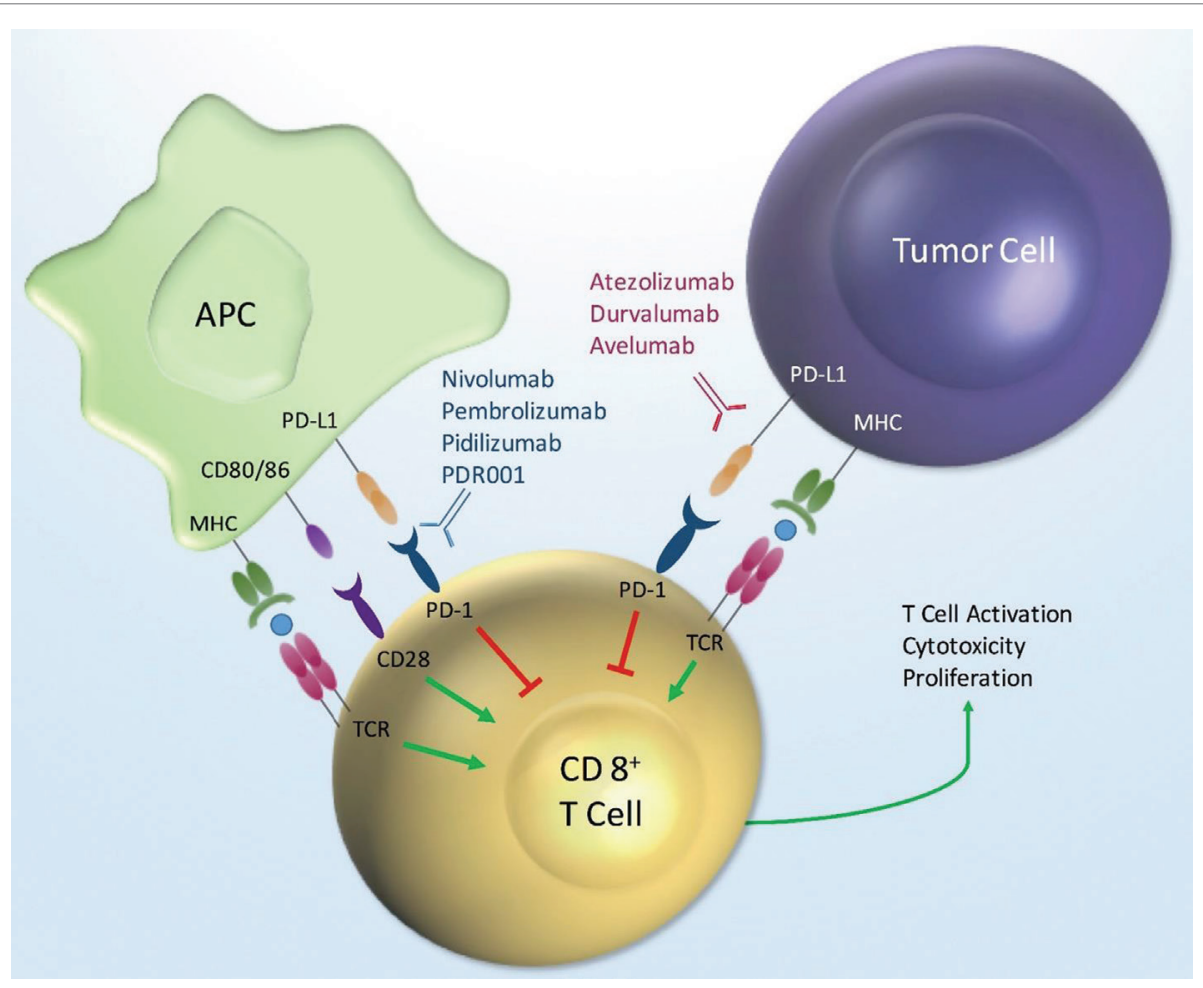

FIGURE 1 | PD-1/PD-L1 mechanism of action. Key: MHC, major histocompatibility; APC, antigen presenting cell; PD-L1, programmed death ligand-1; PD-1, programmed death receptor-1; TCR, T-cell receptor. 
prior progression on treatment with vascular endothelial growth factor receptor tyrosine kinase inhibitors. Eight hundred twentyone patients were enrolled. The median overall survival was 25.0 months (95\% CI, 21.8-not estimable) for nivolumab compared to 19.6 months (95\% CI, 17.6-23.1 months) for everolimus, with a hazard ratio of $0.73(98.5 \% \mathrm{CI}, 057-0.93, P=0.002)$ favoring nivolumab therapy. The objective response rate was 25 versus 5\% (OR 5.98; 95\% CI, 3.68-9.72; $P<0.001$ ) for nivolumab and everolimus, respectively. These results have recently led to approval by the FDA of nivolumab in this setting.

In May 2016, atezolizumab was FDA approved for urothelial carcinoma based on the results of a phase II clinical trial (10). Patients with inoperable locally advanced or metastatic platinumrefractory urothelial carcinoma were treated with atezolizumab $1200 \mathrm{mg}$ every 3 weeks until disease progression or dose-limiting toxicity. Three-hundred ten patients were treated in this singlearm study and stratified by the percent positivity of PD-L1 staining on the tumor infiltrating lymphocytes (Group 1 is $<1 \%$; Groups 2 is $>1 \%$ but $<5 \%$; and Group 3 is $\geq 5 \%$ ). The objective response rates were 15,18 , and $26 \%$, respectively, for groups 1-3. All groups had improved rates of objective responses compared to historic controls (10\%), including a 6-11\% complete response rate.

Based on these encouraging results, and the subsequent FDA approvals, multiple other PD-1 or PD-L1 checkpoint inhibitors are in clinical trials as single-agent therapies or in combination with other antineoplastic therapies including pembrolizumab (PD-1 inhibitor), durvalumab (PD-L1 inhibitor), avelumab (PD-L1 inhibitor), atezolizumab (PD-L1 inhibitor), and PDR001 (PD-1 inhibitor). Here, the authors review the immune-related side effect profiles of PD-1 and PD-L1 inhibitors in GU malignancies.

\section{INCONSISTENCIES IN THE DEFINITION OF IMMUNE-RELATED ADVERSE EVENTS (irAEs) ACROSS PD-1 OR PD-L1 INHIBITOR TRIALS}

Currently, there is no guideline or consensus on how to define and report irAEs in clinical trials. This has resulted in lack of consistency among various clinical trials in reporting the incidence, onset, and duration of AEs. This does create problems when comparing AEs across the trials. For instance, diarrhea and colitis are reported separately in the studies discussed in this review, and the definition for colitis varies between these studies. In the studies with atezolizumab (10-12), irAEs are defined as those events requiring systemic corticosteroids and with no other identifiable underlying cause. By contrast, in one nivolumab study (13), irAEs were defined as any toxicity with a potential immune-mediated etiology, which may or may not have required special monitoring and specific unique interventions. In another nivolumab study (14), reports of irAE were restricted to events requiring use of an immune-modulating therapy, with the exception of endocrine events. In the prescribing information for nivolumab (15) immune-related pneumonitis, nephritis, colitis, and hepatitis were required by definition to have no alternate etiology and necessitated treatment with systemic corticosteroids. The durvalumab trial did not have a formal requirement for systemic corticosteroids, but categorized events as immune-related if the $\mathrm{AE}$ was consistent with an immune-mediated mechanism of action and there was no clear alternate etiology (16). The authors were unable to identify the definition for irAE in the study with pembrolizumab (17). The differences in definitions for irAE among trials involving PD-1 and PD-L1 therapies create hurdles in accurately interpreting the data. There are also other dissimilarities between the reporting of toxicities with irAE and non-immune AEs between these studies. Standardization of the definition of irAE will likely improve the interpretation of clinical trials for clinicians, patients, and policy makers in the future.

\section{METHODS}

A systematic review of the literature was performed according to the Preferred Reporting Items for Systematic Reviews and MetaAnalyses recommendations (18). In October 2016, PubMed, International Pharmaceutical Abstracts (IPA), and EMBASE databases were queried for relevant published literature on all phase II or phase III studies with any PD-1 or PD-L1 therapy in GU malignancies (prostate, urothelial, testicular, and renal cell carcinoma). The search terms may be found in Presentation S1 in Supplementary Material. All database queries were limited to human trials published in the English language. Additionally, the prescribing information for FDA-approved PD-1 or PD-L1 therapies was reviewed. Finally, conference abstracts, posters, and platform presentations from 2016 ESMO and ASCO Meetings were queried for updated published material. Published literature was excluded if the trial involved non-genitourinary malignancies, as well as all phase I studies. Trials involving combination anticancer therapy were excluded; however, concomitant hormonal therapy was allowed. Manufacturers of FDA approved therapies for GU malignancies (nivolumab and atezolizumab) were consulted for Supplementary Material on the outcome of interest when published materials were incomplete.

\section{RESULTS}

\section{Search Results}

A total of 931 citations were identified through EMBASE, 283 citations in PubMed, and 57 abstracts through the IPA. In total, 969 unique studies were identified. After removing the citations for combination trials, published data where outcomes of interest were not reported, trials involving non-genitourinary malignancies and non-primary literature (reviews, guideline updates, etc.), there were eight sources that met criteria for study inclusion (Figure 2).

\section{Atezolizumab}

One clinical trial was identified, with a total of 429 patients with urothelial carcinoma, which evaluated atezolizumab treatment in a single-arm, two cohort study (10-12). Atezolizumab was given as a $1200 \mathrm{mg}$ intravenous infusion every 3 weeks until doselimiting toxicities or until loss of clinical benefit to all patients. 


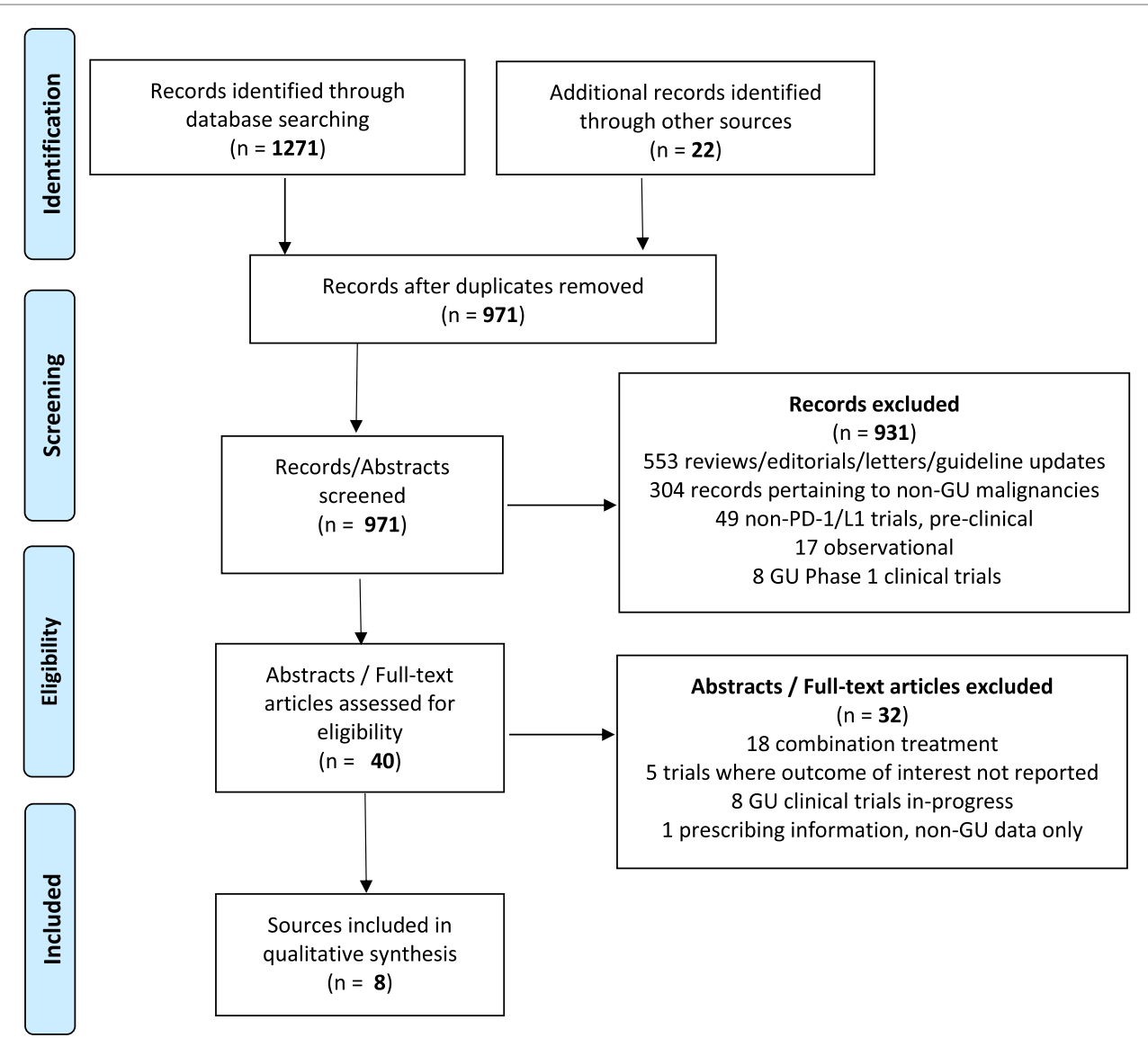

FIGURE 2 | Data selection process (18).

Cohort 1 ( $n=119)$ included patients without prior treatment for metastatic disease and were ineligible for cisplatin-based chemotherapy. Cohort $2(n=310)$ included patients with metastatic disease who progressed in platinum therapy. The irAE incidence was $12 \%$ (14 patients) for all grades, $7 \%$ (8 patients) for grades $3-5$ in cohort 1 (11). This was similar to cohort 2 , with $10 \%$ (31 patients) for all grades and 6\% (19 patients) for grades 3-5 (12). The most common irAEs were rash, transaminitis, rhabdomyolysis, pneumonitis, and hyperbilirubinemia. The most frequent grades 3-5 irAEs were transaminitis and hyperbilirubinemia. Pneumonitis, endocrinopathies, and rhabdomyolysis were noted with $\leq 1 \%$ incidence for grades $3-5$ toxicities (Table 1).

\section{Durvalumab}

One clinical trial was identified, which included 61 patients with urothelial carcinoma who declined, or were ineligible for, or had progressed after platinum-based chemotherapy (16). Durvalumab was given as a $10 \mathrm{mg} / \mathrm{kg}$ intravenous infusion every 2 weeks for up to 12 months. The irAE incidence was 23\% (14 patients) experiencing any grade of toxicity, and 3\% (2 patients) with a grade 3-5 toxicity. The most common irAEs were diarrhea, pruritus, and infusion-related reactions. The two grade 3-5 reactions were biopsy-proven nephritis and an infusion-related reaction (Table $\mathbf{1}$ ).

\section{Nivolumab}

Three clinical trials were identified. In the first study, nivolumab was compared to everolimus in a randomized trial in patients with metastatic renal cell carcinoma who had progressed on one or two antiangiogenic therapies (8). Nivolumab was given as an intravenous infusion at $3 \mathrm{mg} / \mathrm{kg}$ every 2 weeks with a total of 406 patients treated in the nivolumab arm. The overall incidence of all grades of AE rate was $79 \%$, and $19 \%$ of patients experiencing grade 3-4 events. The overall incidence of irAE was not reported. The second study involving 168 metastatic renal cell carcinoma patients tested three different doses of nivolumab in patients who progressed on antiangiogenic therapy. Nivolumab was administered as an intravenous infusion at $0.3,2$, or $10 \mathrm{mg} /$ kg every 3 weeks (13). In the safety population $(n=167)$, the overall incidence of any grade $\mathrm{AE}$ in the $0.3 \mathrm{mg} / \mathrm{kg}$ treatment arms was $75 \%$, and $5 \%$ for grades $3-4$. For the $2 \mathrm{mg} / \mathrm{kg}$ arm, the incidence for any grade was 67\%, with $17 \%$ experiencing grades 3-4 reactions. For the $10 \mathrm{mg} / \mathrm{kg}$ arm, the incidence for all grades was $78 \%$, with $13 \%$ experiencing a grade $3-4$ reaction. Again, the overall incidence of all irAE was not reported. In the third study, nivolumab was given intravenously every 2 weeks at $3 \mathrm{mg} / \mathrm{kg}$ in 78 patients with urothelial carcinoma who previously progressed on platinum-based chemotherapy $(14,23)$. The total number of irAE was not reported. The incidence 
TABLE 1 | Incidence of immune-related adverse events (irAEs) by treatment type for genitourinary (GU) cancers in phase II/III Trials (11-14, 16, 17, 19, 22).

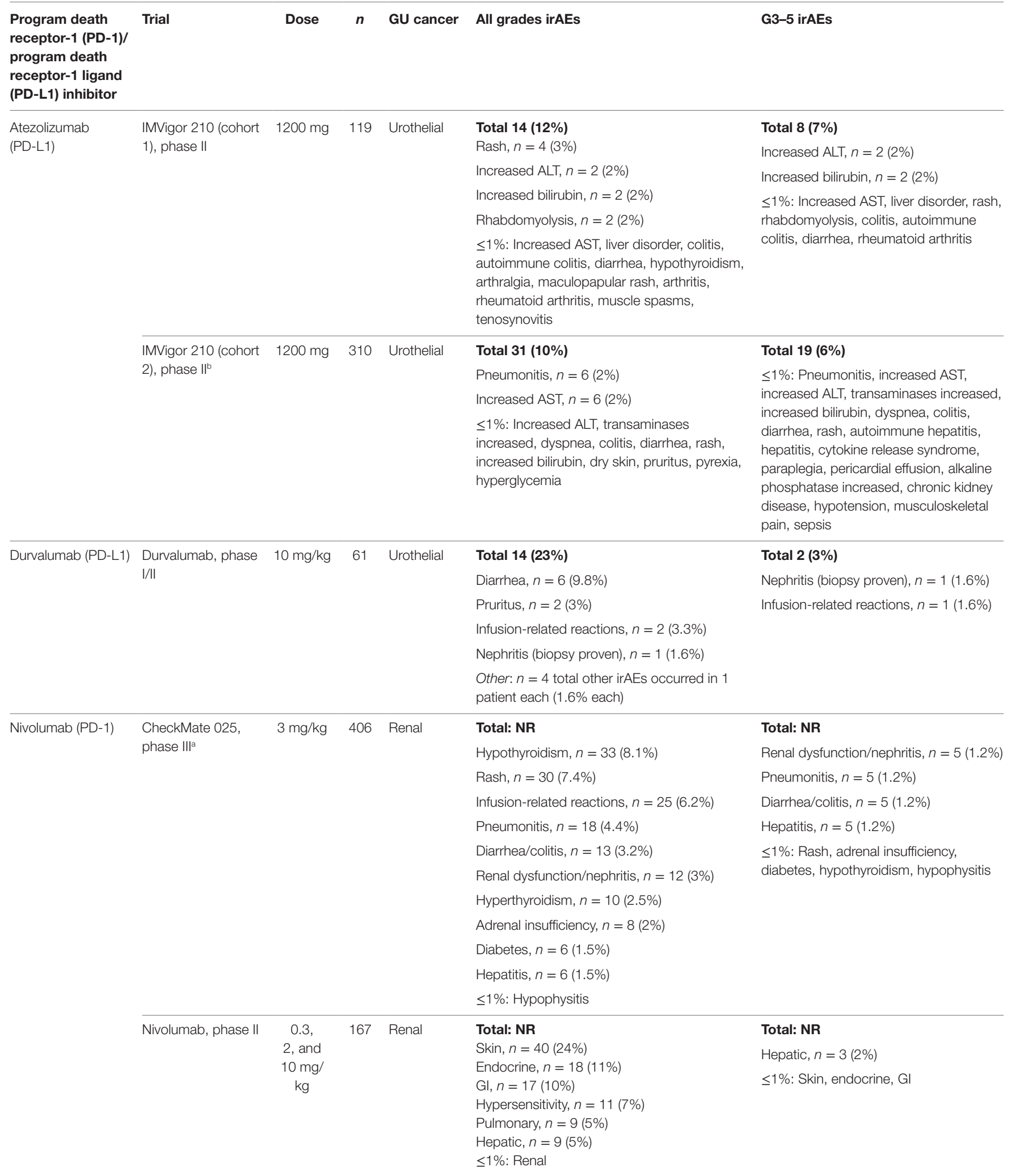




\begin{tabular}{|c|c|c|c|c|c|c|}
\hline $\begin{array}{l}\text { Program death } \\
\text { receptor-1 (PD-1)/ } \\
\text { program death } \\
\text { receptor-1 ligand } \\
\text { (PD-L1) inhibitor }\end{array}$ & Trial & Dose & $n$ & GU cancer & All grades irAEs & G3-5 irAEs \\
\hline & \multirow{8}{*}{$\begin{array}{l}\text { CheckMate 032, } \\
\text { phase I/II }\end{array}$} & \multirow[t]{8}{*}{$3 \mathrm{mg} / \mathrm{kg}$} & \multirow[t]{8}{*}{78} & \multirow[t]{8}{*}{ Urothelial } & Total: NR & Total: NR \\
\hline & & & & & Rash, $n=7$ (9\%) & Hepatitis, $n=3$ (4\%) \\
\hline & & & & & Hypothyroidism/thyroiditis, $n=7$ (9\%) & \multirow{6}{*}{$\begin{array}{l}\leq 1 \% \text { : Pneumonitis (worsened to } \\
\text { G5), diarrhea/colitis, nephritis/renal } \\
\text { impairment, rash }\end{array}$} \\
\hline & & & & & Hyperthyroidism, $n=3$ (4\%) & \\
\hline & & & & & Hepatitis, $n=3$ (4\%) & \\
\hline & & & & & Pneumonitis, $n=2(3 \%)$ & \\
\hline & & & & & Diarrhea/colitis, $n=2(3 \%)$ & \\
\hline & & & & & $\begin{array}{l}\leq 1 \% \text { : Nephritis/renal impairment, } \\
\text { hypersensitivity }\end{array}$ & \\
\hline \multirow{4}{*}{$\begin{array}{l}\text { Pembrolizumab } \\
\text { (PD-1) }\end{array}$} & \multirow{4}{*}{$\begin{array}{l}\text { Pembrolizumab, } \\
\text { phase II }\end{array}$} & \multirow[t]{4}{*}{$200 \mathrm{mg}$} & \multirow[t]{4}{*}{20} & \multirow[t]{4}{*}{ Prostate } & Total $5(25 \%)$ & Total 3 (15\%) \\
\hline & & & & & Colitis, $n=2(10 \%)$ & Colitis, $n=2(10 \%)$ \\
\hline & & & & & Hypothyroidism, $n=2$ (10\%) & Hypothyroidism, $n=1$ (5\%) \\
\hline & & & & & Myositis, $n=1(5 \%)$ & \\
\hline \multirow{4}{*}{$\begin{array}{l}\text { Pembrolizumab } \\
\text { (PD-1) }\end{array}$} & \multirow{4}{*}{$\begin{array}{l}\text { KEYNOTE-052, } \\
\text { phase II }\end{array}$} & \multirow[t]{4}{*}{$200 \mathrm{mg}$} & \multirow[t]{4}{*}{100} & \multirow[t]{4}{*}{ Urothelial } & Total: NR & Total: NR \\
\hline & & & & & Hypothyroidism, $n=6$ (6\%) & Pneumonitis, $n=2(2 \%)$ \\
\hline & & & & & Pneumonitis, $n=3(3 \%)$ & $\leq 1 \%$ : Nephritis, colitis \\
\hline & & & & & $\leq 1 \%$ : Nephritis, colitis & \\
\hline
\end{tabular}

${ }^{a}$ CheckMate 025: permission obtained to use data from nivolumab website: $h$ ttp://www.opdivoyervoyhcp.com/servlet/servlet.FileDownload?file=00PiO00000SRPWfEAP. bMvigor 210 (cohort 2): reported all grades irAEs occurring in $\geq 2$ patients, reported grades 3-4 irAEs occurring in $\geq 1$ patient.

for any $\mathrm{AD}$ for all grades was $81 \%$ with $22 \%$ of patients experiencing grade 3 events. Notably, 1 patient experienced a grade 5 event (pneumonitis, patient died). Pneumonitis, colitis, transaminitis, hypothyroidism, and rash were the most commonly reported grades 3-4 irAEs. Hypophysitis, adrenal insufficiency, diabetes mellitus, hyperthyroidism, and nephritis were also reported (Table 1).

\section{Pembrolizumab}

Pembrolizumab, a PD-1 antagonist, was given as a 200-mg flat dose intravenous infusion every 3 weeks for four doses in patients with metastatic castration-resistant prostate cancer, previously progressing on enzalutamide (17). Patients could not have received prior chemotherapy. There were 20 patients treated on this study. Any grade of irAE occurred in 25\% of patients (5 patients) and grade 3 irAE occurred in 15\% of patients ( 3 patients). The most common irAEs of any grade were myositis $(n=1)$, hypothyroidism $(n=2)$, and colitis $(n=2)$. The three grade 3 events were hypothyroidism $(n=1)$ and colitis $(n=2)$. There were no grade 4 or 5 events reported (Table 1 ).

The KEYNOTE-052 study (19) in cisplatin-ineligible patients evaluated pembrolizumab in the first-line setting in urothelial carcinoma in 200 patients. Pembrolizumab was given as $200 \mathrm{mg}$ IV every 3 weeks. The treatment related AE for any grade was 67 and $15 \%$ for grades $3-4$ events. The overall incidence for irAE was not reported. Pneumonitis was noted in 3\% of patients, nephritis in $1 \%$, colitis in $1 \%$, and hypothyroidism in $6 \%$.
TABLE 2 | Comparison of selected immune-related adverse event (irAE) in GU versus non-genitourinary clinical trials $(11-17,19-22)$.

\begin{tabular}{lcccc}
\hline Adverse event & $\begin{array}{c}\text { Incidence, } \\
\text { any grade } \\
\text { (GU only } \\
\text { trials) (\%) }\end{array}$ & $\begin{array}{c}\text { Incidence, } \\
\text { grades 3-5 } \\
\text { (GU only } \\
\text { trials) (\%) }\end{array}$ & $\begin{array}{c}\text { Incidence } \\
\text { any grade } \\
\text { (non-GU } \\
\text { clinical } \\
\text { trials) (\%) }\end{array}$ & $\begin{array}{c}\text { Incidence, } \\
\text { grades 3-5 } \\
\text { (non-GU } \\
\text { clinical trials) } \\
\text { (\%) }\end{array}$ \\
\hline $\begin{array}{l}\text { Hypothyroid/ } \\
\text { thyroiditis }\end{array}$ & $0.8-9$ & $0-0.6$ & $3.9-12$ & $0-0.1$ \\
$\begin{array}{l}\text { Diabetes/DKA } \\
\text { LFT changes/ }\end{array}$ & $0-1.5$ & $0-0.7$ & $0.8-0.8$ & $0.4-0.7$ \\
$\begin{array}{l}\text { hepatitis } \\
\text { Pneumonitis }\end{array}$ & $1.5-5.4$ & $1-3.8$ & $0.3-3.4$ & $0.3-2.7$ \\
$\begin{array}{l}\text { Encephalitis } \\
\text { Colitis/diarrhea }\end{array}$ & N-4.4 & $0-2$ & $1.8-3.5$ & $0.25-1.9$ \\
$\begin{array}{l}\text { Hypophysitis } \\
\text { Renal Dysfunction/ }\end{array}$ & $0.3-1.6$ & NR & $0.2-0.8$ & $0.0-0.2$ \\
nephritis & $0-0.5$ & $0-0.2$ & $0.2-0.9$ & $0.2-0.4$ \\
Myositis $^{\text {a }}$ & $0.8-5$ & $0-0.8$ & $0.3-4.9$ & $0.0-0.5$ \\
\hline
\end{tabular}

GU, genitourinary; DKA, diabetic ketoacidosis; LFT, liver function test; NR, not reported. aReported in only one study.

The irAEs reported in all of these studies with GU cancers, including the incidence of grades 3-5 toxicities, are similar to those reported in trials of PD-1 inhibitors in non-genitourinary clinical trials. Thus, the incidence of irAE in GU cancers appears to be representative of the overall cancer patient population (Table 2). 


\section{UNDERREPORTING OF AEs}

Recent analysis of clinical trials involving immune checkpoint therapy, including PD-1 and PD-L1 therapies, highlights the potential concern of underreporting of irAEs. Chen et al (24) evaluated all clinical trials involving any checkpoint inhibitor and noted large variability in the quality of irAE reporting. The authors used a 21-point oncology specific AE reporting score based on the CONSORT criteria as the quality benchmark. The median incidence of grades 3-4 toxicities was $22 \%$, however, with a range of $0-66 \%$. The review identified overall poor reporting of toxicity management (8\%), reversibility of $\mathrm{AE}(6 \%)$, and the onset of the AE (14\%). These findings are similar to an updated analysis that was reported this year. Bossi et al. (25) analyzed immunotherapy and targeted therapy clinical trials for the quality of AE reporting, also benchmarked against the CONSORT criteria. More than $90 \%$ of clinical trials scored poorly in their reporting of recurrent and late toxicities, and in the duration of the AEs. This is a consistent finding between these two analyses. Most trials did not report on the time to the AE occurrence (86\%), and only reported on AEs that occurred at a frequency above a fixed threshold (75\%). Dose reductions due to $\mathrm{AE}$ were not reported in one-third of trials.

If studies are only reporting irAE at a specific threshold, or not fully reporting on the extent of the disease complications, then the potential risk with this therapeutic class might be significantly underappreciated. This issue is particularly relevant given the diversity in reported AEs and the generally low incidence of any one specific irAE from PD-1/PD-L1 therapies. In aggregate though, the overall incidence of any significant (grades 3-4) irAE is relatively frequent $(16-22 \%)$ as reported in the clinical trials examined in this review. This is consistent with the reported grades 3-4 AE rate in clinical trials across all cancers (24).

All the reported evidence to date of PD-1 and PD-L1 inhibitors in GU malignancies are based on clinical trials. There are no reports of AEs from a real-world experience. This distinction is important as the complication rates may be higher in general practice compared to the clinical trial experience (26-28). However, some studies document comparable incidences of AEs between clinical trials and the real-world experience $(29,30)$. It is possible that the incidence of irAE and $\mathrm{AE}$ will be higher for PD-1 and PD-L1 inhibitors than is reported in these clinical trials. This increased incidence could be related to less experience with the safe use of these agents between study investigators and community practice providers given the unique type of irAEs, the strict inclusion/exclusion criteria of clinical trials and the rare or delayed nature of the irAEs.

Familiarity with the complications of treatment from immunotherapy is important for all oncologists. The indications for these therapies are continuing to expand, and use of these treatments is expected to continue to grow rapidly over the next few years. Currently, there are three FDA-approved PD-1 or PD-L1 agents [nivolumab, pembrolizumab, and atezolizumab $(15,20,21)$ ], two of which have indications for use in GU malignancies (nivolumab and atezolizumab). In contrast, there are over 500 clinical trials in all cancers (31). Likely, some of these newer agents will obtain FDA approval, and the number of clinical indications will expand. Treating oncologists will likely be using these therapies more frequently and thereby, encounter the irAEs frequently. This is a rapidly evolving area in GU oncology with many new clinical trials opening. New results are constantly being published in the literature and presented at professional meetings each year. With many novel therapies and new immunotherapy combinations being studied, it will remain important to be aware of the incidence and prevalence of irAEs in GU malignancies.

\section{TREATMENT OF irAE}

Treatment of any irAE caused by PD-directed therapies is largely determined by the severity as defined in the Common Terminology Criteria for Adverse Events (CTCAE) criteria. This validated tool is not specifically designed for immunotherapy, leading some people to question the utility of this tool (24). The CTCAE does not adequately capture the duration of therapy or the irreversibility of an $\mathrm{AE}$, both of which are important considerations in treatment planning. The CTCAE may be modified in the future to better reflect the specific complications seen with immunotherapy. Still, this is a reasonable tool in identifying the severity of illness and therefore guiding the clinician in taking the appropriate steps for initiating treatment.

With all AEs encountered during immunotherapy treatment, it is important to rule out other causes in all cases. Frequently, multidisciplinary treatment is required for both a thorough workup and appropriate treatment, especially regarding serious (grades 3-4) irAEs. Oftentimes, this approach is most effectively performed at referral centers with subspecialty care. The majority of serious AEs are reversible with discontinuation of immune therapy and appropriate treatment. For that reason, corticosteroid therapy generally can be safely tapered over approximately 1 month after the symptoms begin to improve. Endocrinopathies are a notable exception, as these AEs can be permanent, potentially requiring life-long therapy $(32,33)$.

Treatment algorithms developed previously for the management of ipilimumab therapy have been reported $(34,35)$. There are no trials or reported experiences evaluating treatment approaches specifically for PD-1- or PD-L1-mediated irAEs. The treatment algorithm for all checkpoint blockade therapy is based on the experience with ipilimumab (22).

\section{LIMITATIONS}

In addition to the issue of inconsistent reporting of the irAEs in these trials, as described above, we were unable to find published data that met the inclusion criteria of phase II or phase III studies for avelumab (PD-L1 antagonist), PDR001 (PD-1 antagonist), and pidilizumab (presumed PD-1 antagonist). In addition, the sample size was small for durvalumab. Because the reported irAEs are similar between all the PD-1 and PD-L1 antagonists reported to date, the AEs and irAEs discussed here appear to be a drug class effects and not simply related to a single compound. Therefore, we expect that other PD-1 and PD-L1 directed therapies, such as pidilizumab, are likely to have a similar incidence of irAEs. Given the consistency of the toxicity profile with GU malignancies compared to other malignancies, the immune-mediated events do not appear to be significantly related to disease type. 
The Keynote-045 study was recently reported at the SITC meeting in November 2016 (after the date cutoff in this systematic review). This study provides additional safety information related to pembrolizumab. This trial includes an additional 270 patients treated with pembrolizumab. When published, these data will provide significantly more safety data for this agent in urothelial cancers.

\section{CONCLUSION}

Overall, the incidence of serious (grades 3-4) AEs was noted in approximately $3-15 \%$ of GU cancer patients treated in these phase II and III monotherapy trials of PD-1 or PD-L1 checkpoint inhibitors. The reporting for $\mathrm{AE}$ and irAE was different between the studies, limiting the accuracy of identifying the true incidence for irAE. Although, the most common organ sites of toxicity include the skin, endocrine organs, lung, and the gastrointestinal tract, any organ can be affected. The AEs noted were similar between all the agents tested, highlighting the overall class effect of these therapies. The irAEs seen in patients with GU cancers were similar to those seen in patients with other cancers. It is well established that incidence of irAEs with checkpoint inhibitors in the clinical trials is underreported.

\section{REFERENCES}

1. Weinstock M, McDermott D. Targeting PD-1/PD-L1 in the treatment of metastatic renal cell carcinoma. Ther Adv Urol (2015) 7(6):365-77. doi:10.1177/1756287215597647

2. Sunshine J, Taube JM. PD-1/PD-L1 inhibitors. Curr Opin Pharmacol (2015) 23:32-8. doi:10.1016/j.coph.2015.05.011

3. Carosella ED, Ploussard G, LeMaoult J, Desgrandchamps F. A systematic review of immunotherapy in urologic cancer: evolving roles for targeting of CTLA-4, PD-1/PD-L1, and HLA-G. Eur Urol (2015) 68(2):267-79. doi:10.1016/j.eururo.2015.02.032

4. Bracarda S, Altavilla A, Hamzaj A, Sisani M, Marrocolo F, Del Buono $\mathrm{S}$, et al. Immunologic checkpoints blockade in renal cell, prostate, and urothelial malignancies. Semin Oncol (2015) 42(3):495-505. doi:10.1053/j. seminoncol.2015.02.004

5. Tsiatas M, Grivas P. Immunobiology and immunotherapy in genitourinary malignancies. Ann Transl Med (2016) 4(14):270. doi:10.21037/atm.2016.06.29

6. Yamazaki T, Akiba H, Iwai H, Matsuda H, Aoki M, Tanno Y, et al. Expression of programmed death 1 ligands by murine T cells and APC. J Immunol (2002) 169(10):5538-45. doi:10.4049/jimmunol.169.10.5538

7. Thompson RH, Gillett MD, Cheville JC, Lohse CM, Dong H, Webster WS, et al. Costimulatory molecule $\mathrm{B} 7-\mathrm{H} 1$ in primary and metastatic clear cell renal cell carcinoma. Cancer (2005) 104(10):2084-91. doi:10.1002/cncr.21470

8. Motzer RJ, Escudier B, McDermott DF, George S, Hammers HJ, Srinivas S, et al. Nivolumab versus everolimus in advanced renal-cell carcinoma. $N$ Engl J Med (2015) 373(19):1803-13. doi:10.1056/NEJMoa1510665

9. McDermott DF, Drake CG, Sznol M, Choueiri TK, Powderly JD, Smith DC, et al. Survival, durable response, and long-term safety in patients with previously treated advanced renal cell carcinoma receiving nivolumab. J Clin Oncol (2015) 33(18):2013-20. doi:10.1200/JCO.2014.58.1041

10. Rosenberg JE, Hoffman-Censits J, Powles T, van der Heijden MS, Balar AV, Necchi A, et al. Atezolizumab in patients with locally advanced and metastatic urothelial carcinoma who have progressed following treatment with platinum-based chemotherapy: a single-arm, multicentre, phase 2 trial. Lancet (2016) 387(10031):1909-20. doi:10.1016/S0140-6736(16)00561-4

11. Bellmunt J, Balar AV, Galsky MD, Loriot Y, Theodore C, Grande E, et al. IMvigor210: updated analyses of first-line atezolizumab in cisplatin-ineligible locally advanced/metastatic urothelial carcinoma. Ann Oncol (2016) 27(Suppl 6):abstract 782D. doi:10.1093/annonc/mdw373.10
Additionally, the real-world use of these drugs is likely going to be widespread, and the real-world patients likely will be frailer with more comorbidities than the clinical trial patients. This suggests that the overall magnitude of the irAEs of PD-1 and PD-L1 inhibitors are expected to be much higher in the realworld setting. Increased awareness, and timely recognition and management of these toxicities are expected to reduce risk and improve clinical benefit with this class of agents.

\section{AUTHOR CONTRIBUTIONS}

$\mathrm{BM}$ and $\mathrm{EB}$ contributed equally to the preparation of this manuscript and compiled the database search for this project. BM, EB, DG, and NA wrote the manuscript and made revisions to the manuscript, tables, and figures. BM, EB, and DG created tables and figures.

\section{SUPPLEMENTARY MATERIAL}

The Supplementary Material for this article can be found online at http://journal.frontiersin.org/article/10.3389/fonc. 2017.00056/full\#supplementary-material.

12. Dreicer R, Hoffman-Censits J, Flaig T, Grande E, Balmanoukian A, von Amsberg G, et al. Updated efficacy from IMvigor210: atezolizumab in platinum-treated locally advanced/metastatic uothelial carcinoma (mUC). J Clin Oncol (2016) 34(Suppl):abstract 4515 and oral presentation.

13. Motzer RJ, Rini BI, McDermott DF, Redman BG, Kuzel TM, Harrison MR, et al. Nivolumab for metastatic renal cell carcinoma: results of a randomized phase II trial. J Clin Oncol (2015) 33(13):1430-7. doi:10.1200/JCO.2014.59.0703

14. Sharma P, Callahan MK, Bono P, Kim J, Spiliopoulou P, Calvo E, et al. Nivolumab monotherapy in recurrent metastatic urothelial carcinoma (CheckMate 032): a multicentre, open-label, two-stage, multi-arm, phase 1/2 trial. Lancet Oncol (2016) 17(11):1590-8. doi:10.1016/S1470-2045(16)30496-X

15. OPDIVO \{package insert\}. Princeton, NJ: Bristol-Myers Squibb Company (2017). Available from: http://packageinserts.bms.com/pi/pi_opdivo.pdf

16. Massard C, Gordon MS, Sharma S, Rafii S, Wainberg ZA, Luke J, et al. Safety and efficacy of durvalumab (MEDI4736), an anti-programmed cell death ligand-1 immune checkpoint inhibitor, in patients with advanced urothelial bladder cancer. J Clin Oncol (2016) 34(26):3119-25. doi:10.1200/JCO.2016. 67.9761

17. Graff JN, Alumkal JJ, Drake CG, Thomas GV, Redmond WL, Farhad M, et al. First evidence of significant clinical activity of PD-1 inhibitors in metastatic castration resistant prostate cancer (mCRPC). Ann Oncol (2016) 27(Suppl 6):abstract 719O. doi:10.1093/annonc/mdw372.03

18. Moher D, Liberati A, Tetzlaff J, Altman DG, PRISMA Group. Preferred reporting items for systematic reviews and meta-analyses: the PRISMA statement. Ann Intern Med (2009) 151(4):264-9. doi:10.7326/0003-4819-151-4-200908180-00135

19. Balar AVBJ, O’Donnell PH, Castellano D, Grivas P, Vuky J, Powles T, et al. First-line pembrolizumab in cisplatin-ineligible unresectable or metastatic urothelial cancer: interim results for KEYNOTE-052. Presented at: 2016 ESMO Congress; October 7-11, 2016; Copenhagen, Denmark (2016).

20. Tecentriq \{package insert\}. South San Francisco, CA: Genetech, Inc (2016). Available from: https://www.gene.com/download/pdf/tecentriq_prescribing. pdf

21. Keytruda \{package insert\}. Whitehouse Station, NJ: Merck \& Co., Inc. (2016). Available from: http://www.merck.com/product/usa/pi_circulars/k/ keytruda/keytruda_pi.pdf

22. Immune-Mediated Adverse Reactions Management Guide. (2017). Available from: http://www.opdivohcpinfo.com/servlet/servlet.FileDownload?file $=00$ Pi000000Hj19REAR 
23. Sharma P, Bono P, Kim JW, Spiliopoulou P, Calvo P, Pillai RN, et al. Efficacy and safety of nivolumab monotherapy in metastatic urothelial cancer (mUC): results from the phase I/II CheckMate 032 study. J Clin Oncol (2016) 34(Suppl):abstr 4501.

24. Chen TW, Razak AR, Bedard PL, Siu LL, Hansen AR. A systematic review of immune-related adverse event reporting in clinical trials of immune checkpoint inhibitors. Ann Oncol (2015) 26(9):1824-9. doi:10.1093/annonc/ mdv182

25. Bossi P, Botta L, Bironzo P, Sonetto C, Musettini G, Sbrana A, et al. Systematic review of adverse events reporting in clinical trials leading to approval of targeted therapy and immunotherapy. Ann Oncol (2016) 27(Suppl 6):Abstract 320. doi:10.1093/annonc/mdw366.05

26. Elliott L, Fidler C, Ditchfield A, Stissing T. Hypoglycemia event rates: a comparison between real-world data and randomized controlled trial populations in insulin-treated diabetes. Diabetes Ther (2016) 7(1):45-60. doi:10.1007/ s13300-016-0157-z

27. Asche CV, McAdam-Marx C, Shane-McWhorter L, Sheng X, Plauschinat CA. Evaluation of adverse events of oral antihyperglycemic monotherapy experienced by a geriatric population in a real-world setting: a retrospective cohort analysis. Drugs Aging (2008) 25(7):611-22. doi:10.2165/00002512200825070-00006

28. Seruga B, Templeton AJ, Badillo FE, Ocana A, Amir E, Tannock IF. Underreporting of harm in clinical trials. Lancet Oncol (2016) 17(5):e209-19. doi:10.1016/S1470-2045(16)00152-2

29. Wang C, Cho B, Xiao D, Wajsbrot D, Park PW. Effectiveness and safety of varenicline as an aid to smoking cessation: results of an inter-Asian observational study in real-world clinical practice. Int J Clin Pract (2013) 67(5):469-76. doi:10.1111/ijcp.12121

30. Antinori A, Borderi M, Cauda R, Bini T, Chirianni A, Squillace $\mathrm{N}$, et al. Safety of darunavir/ritonavir (DRV/r) in HIV-1-infected
$\mathrm{DRV} / \mathrm{r}$-experienced and -naive patients: analysis of data in the real-world setting in Italy. J Int AIDS Soc (2014) 17(4 Suppl 3):19573. doi:10.7448/IAS.17. 4.19573

31. Goodman N. Too many PD-1 trials for adults, too few for children. Cancer Lett (2016). Available from: http://cancerletter.com/articles/20161014_3/

32. Tanaka R, Fujisawa Y, Maruyama H, Nakamura Y, Yoshino K, Ohtsuka M, et al. Nivolumab-induced thyroid dysfunction. Jpn J Clin Oncol (2016) 46(6):575-9. doi:10.1093/jjco/hyw036

33. Johnson DB, Friedman DL, Berry E, Decker I, Ye F, Zhao S, et al. Survivorship in immune therapy: assessing chronic immune toxicities, health outcomes, and functional status among long-term ipilimumab survivors at a single referral center. Cancer Immunol Res (2015) 3(5):464-9. doi:10.1158/23266066.CIR-14-0217

34. Fecher LA, Agarwala SS, Hodi FS, Weber JS. Ipilimumab and its toxicities: a multidisciplinary approach. Oncologist (2013) 18(6):733-43. doi:10.1634/ theoncologist.2012-0483

35. Postow MA. Managing immune checkpoint-blocking antibody side effects. Am Soc Clin Oncol Educ Book (2015) 2015:76-83. doi:10.14694/ EdBook_AM.2015.35.76

Conflict of Interest Statement: The authors declare that the research was conducted in the absence of any commercial or financial relationships that could be construed as a potential conflict of interest.

Copyright (c) 2017 Maughan, Bailey, Gill and Agarwal. This is an open-access article distributed under the terms of the Creative Commons Attribution License (CC BY). The use, distribution or reproduction in other forums is permitted, provided the original author(s) or licensor are credited and that the original publication in this journal is cited, in accordance with accepted academic practice. No use, distribution or reproduction is permitted which does not comply with these terms. 